\title{
Effects of impulsive noise on marine mammals: investigating range-dependent risk
}

\author{
Gordon Hastie, ${ }^{1,5}$ Nathan D. Merchant, ${ }^{2}$ Thomas Götz, ${ }^{1}$ Debbie J. F. Russell,${ }^{1,3}$ Paul Thompson, ${ }^{4}$ and \\ VINCENT M. JANIK ${ }^{1}$ \\ ${ }^{1}$ Sea Mammal Research Unit, Scottish Oceans Institute, University of St Andrews, St Andrews KY16 8 LB United Kingdom \\ ${ }^{2}$ Centre for Environment, Fisheries and Aquaculture Science, Pakefield Road, Lowestoft NR33 OHT United Kingdom \\ ${ }^{3}$ Centre for Research into Ecological and Environmental Modelling, The Observatory, University of St Andrews, St Andrews \\ KY16 9LZ United Kingdom \\ ${ }^{4}$ Lighthouse Field Station, Institute of Biological and Environmental Sciences, University of Aberdeen, George Street, Cromarty \\ IV11 8 YL United Kingdom
}

Citation: Hastie, G., N. D. Merchant, T. Götz, D. J. F. Russell, P. Thompson, and V. M. Janik. 2019. Effects of impulsive noise on marine mammals: investigating range-dependent risk. Ecological Applications 29(5):e01906. 10.1002/eap.1906

Abstract. Concerns exist about the impacts of underwater noise on marine mammals. These include auditory damage, which is a significant risk for marine mammals exposed to impulsive sounds such as explosions, pile-driving, and seismic air guns. Currently, impact assessments use different risk criteria for impulsive and non-impulsive sounds (e.g., ships, drilling). However, as impulsive sounds dissipate through the environment, they potentially lose hazardous features (e.g., sudden onset) and become non-impulsive at some distance from the source. Despite management implications, a lack of data on range-dependent characteristics currently limits their inclusion in impact assessments. We address this using acoustic recordings of seismic air guns and pile-driving to quantify range dependency in impulsive characteristics using four criteria: (1) rise time $<25 \mathrm{~ms}$; (2) quotient of peak pressure and pulse duration $>5,000 \mathrm{~Pa} / \mathrm{s}$; (3) duration $<1 \mathrm{~s}$; (4) crest factor $>15 \mathrm{~dB}$. We demonstrate that some characteristics changed markedly within ranges of $\sim 10 \mathrm{~km}$, and that the mean probability of exceeding criteria 1 and 2 was $<0.5$ at ranges $>3.5 \mathrm{~km}$. In contrast, the mean probability of exceeding criteria 3 remained $>0.5$ up to $\sim 37.0 \mathrm{~km}$, and the mean probability of exceeding criteria 4 remained $<0.5$ throughout the range. These results suggest that a proportion of the recorded signals should be defined as impulsive based on each of the criteria, and that some of the criteria change markedly as a result of propagation. However, the impulsive nature of a sound is likely to be a complex interaction of all these criteria, and many other unrelated parameters such as duty cycle, recovery periods, and sound levels will also strongly affect the risk of hearing damage. We recommend future auditory damage studies and impact assessments explicitly consider the ranges at which sounds may lose some of their potentially hazardous characteristics.

Key words: auditory damage; marine mammals; pile driving; seismic survey; sound propagation; underwater noise.

\section{INTRODUCTION}

Impulsive sounds from human-made sources occur widely in the marine environment; these are produced intentionally (e.g., seismic surveys or sonar) or occur as a by-product of an activity (e.g., explosives or pile driving) and are some of the most powerful sounds produced underwater (Gordon et al. 2004). They are also likely to become more widespread in the coming years as the petroleum industry looks to undertake surveys in new offshore areas and pile driving associated with offshore

Manuscript received 30 May 2018; revised 9 January 2019; accepted 19 February 2019. Corresponding Editor: Eric J. Ward.

${ }^{5}$ E-mail: gdh10@st-andrews.ac.uk wind turbine construction is carried out to meet ambitious renewable energy targets in many countries.

Exposure to these sounds has raised concerns about potential impacts on marine wildlife (in particular marine mammals). The mammalian auditory system is known to be vulnerable to damage from intensive impulsive sounds (Henderson and Hamernik 1986, Kryter 1994, Finneran et al. 2000, 2002, Yost 2000), and studies of terrestrial mammals generally conclude that, at comparable sound levels, exposure to impulsive sound is more hazardous than non-impulsive sound with respect to the onset, growth, and recovery of hearing damage (Ward et al. 1959, Fletcher 1970, Luz and Hodge 1970, Buck 1982, Sulkowski and Lipowczan 1982, Hamernik et al. 1987, Dunn et al. 1991).

This greater sensitivity to impulsive sound (with respect to hearing damage) is reflected in sound 
exposure risk criteria for marine mammals where lower sound exposure level (SEL) values are specified for impulsive noise (Southall et al. 2007, National Marine Fisheries Service 2018, Southall et al. 2019). These studies developed a series of weighting curves based on the hearing characteristics of marine mammal species groups and reviewed auditory damage studies to provide exposure criteria for both impulsive and non-impulsive underwater sounds. Critically, both studies predict that, for marine mammals exposed to impulsive sounds, the onset of permanent auditory damage (permanent threshold shift, PTS) would occur at lower weighted cumulative sound exposure levels (cSELs) than for exposure to non-impulsive sounds (Southall et al. 2007, National Marine Fisheries Service 2018, Southall et al. 2019).

Impulsive sounds can be defined in many ways (Henderson and Hamernik 1986, Starck and Pekkarinen 1987, Thiery 1987). In broad terms, they are described as brief, broadband (i.e., extend across a wide range of frequencies), atonal, and transient, characterized by a relatively rapid rise time from onset to maximal sound pressure (Southall et al. 2007). As described above, the features of an impulsive sound are important when considering auditory damage in marine mammals. However, as Southall et al. (2007) highlight, a sound that has impulsive characteristics at the source may, as a result of propagation effects, lose those characteristics (e.g., duration, rise time) and could potentially be characterized as a non-impulsive sound at some (variable) distance from source.

This has important implications for the prediction of the potential impacts of offshore activities during the environmental assessment process. Specifically, the thresholds for PTS onset as a result of exposure to impulsive signals underwater (186 dB re: $1 \mu \mathrm{Pa}^{2}-\mathrm{s}$ for pinnipeds and $198 \mathrm{~dB}$ re: $1 \mu \mathrm{Pa}^{2}-\mathrm{s}$ for cetaceans) are lower than the non-impulsive (203 $\mathrm{dB}$ re: $1 \mu \mathrm{Pa}^{2}-\mathrm{s}$ for pinnipeds and $215 \mathrm{~dB}$ re: $1 \mu \mathrm{Pa}^{2}-\mathrm{s}$ for cetaceans) thresholds (Southall et al. 2007). The estimated risks of auditory damage could therefore be overestimated in cases where impulsive signals become non-impulsive as a result of propagation. This issue was highlighted recently during the development of noise exposure guidance, which investigated the transition between impulsive and non-impulsive sound with range from the source (National Marine Fisheries Service 2015). At a draft stage, this guidance attempted to formalize the range at which impulsive sounds transition to having physical characteristics less likely to result in auditory injury (National Marine Fisheries Service 2015). However, this approach did not appear in the final guidance (National Marine Fisheries Service 2018, Southall et al. 2019) due to a lack of evidence to identify where this transition occurs. The authors highlight that a better understanding how potentially injurious characteristics of a sound change under various propagation conditions is required to inform the development and application of appropriate thresholds. Further, Southall et al. (2019) note that the respective exposure criteria (impulsive or non-impulsive) should be applied based on signal features likely to be received by animals rather than by signal features at the sound source. Here, we directly address this data gap by describing the acoustic signals recorded in the vicinity of seismic air guns and pile driving and measure how their characteristics change with range from source. We then explore the implications of this on predictions of auditory damage in marine mammals.

\section{Methods}

We investigated changes in the characteristics of underwater impulsive sounds with range from source, using a series of underwater recordings made during pile driving and seismic surveys in the North Sea.

\section{Recordings: pile driving}

Recordings of the signals from pile driving were made in 2006 and 2012 during the installation of offshore wind turbine foundations at two locations; the Wash, SE England (during 2012), and the Moray Firth, NE Scotland (during 2006). Pile driving in the Wash was carried out on a submerged sandbank (water depths $\sim 8-20 \mathrm{~m}$ ) approximately $8 \mathrm{~km}$ off the coast of southeast England $\left(53^{\circ} 11.5^{\prime} \mathrm{N}, 0^{\circ} 29.5^{\prime} \mathrm{E}\right)$. Recordings of 704 piling signals were made at a depth of approximately $1 \mathrm{~m}$ from a boat at locations between 1 and $9.5 \mathrm{~km}$ from the pile driving; further details of the pile driving and the recordings are provided in Hastie et al. (2015) and Appendix S1. Pile driving in the Moray Firth $\left(58^{\circ} 06^{\prime} \mathrm{N}, 03^{\circ} 04^{\prime} \mathrm{W}\right)$ was carried out in 2006 approximately $25 \mathrm{~km}$ from the nearest coastline and in a water depth of $42 \mathrm{~m}$. Recordings of 699 piling signals were made at a depth of approximately $5 \mathrm{~m}$ between 0.5 and $41 \mathrm{~km}$ from the pile-driving at approximately $2 \mathrm{~km}$ intervals; further details are provided in Bailey et al. (2010) and Appendix S1.

\section{Recordings: seismic survey}

Seismic surveys were conducted over $10 \mathrm{~d}$ in 2011 within the Moray Firth, northeast Scotland, using a 470 cubic inch $\left(1\right.$ cubic inch $\left.=16.39 \mathrm{~cm}^{3}\right)$ air gun array with a shot point interval of 5-6 s. Water depths in the study area were typically less than $50 \mathrm{~m}$. Recordings of the 2,166 air gun signals were made at a depth of $10 \mathrm{~m}$ from a boat between 1.6 and $61.8 \mathrm{~km}$ from the seismic survey vessel; further details of the seismic surveys and the recordings are provided in Thompson et al. (2013a) and Appendix S1.

\section{Acoustic metric analysis}

For each recorded seismic and pile driving signal, we measured a series of acoustic characteristics; these were range from source $(\mathrm{m})$, rise time (ms), pulse duration (s), peak pressure $(\mathrm{Pa})$, and the crest factor $(\mathrm{dB})$. For a more detailed description of the calculation of each of these characteristics, see Appendix S2. All characteristics were measured using MATLAB (v2015; The MathWorks, 
Natick, Massachusetts, USA). With specific reference to the influence of potential changes in signal characteristics on predictions of auditory injury, we also calculated the quotient of peak pressure $(\mathrm{Pa})$ and pulse duration (s). This is based on analyses suggesting that a quotient of 5,000 is a precautionary approximation of where most impulsive sound sources begin to transition to having physical characteristics less likely to result in auditory injury (National Marine Fisheries Service 2015).

We investigated the relationship between distance from source and a selection of the acoustic characteristics previously used to define impulses: signal duration and rise time (Southall et al. 2007), crest factor (Starck and Pekkarinen 1987), and the quotient of peak pressure (Pa) and pulse duration (s; National Marine Fisheries Service 2015). For each characteristic, the acoustic signals were labelled as impulsive or non-impulsive based on predefined impulsive thresholds: signal duration less than $1 \mathrm{~s}$ (Southall et al. 2007, National Marine Fisheries Service 2015 , 2016), rise time $<25 \mathrm{~ms}$, crest factor greater than $15 \mathrm{~dB}$ (Starck and Pekkarinen 1987), or a quotient of peak pressure $(\mathrm{Pa})$ and pulse duration $(\mathrm{s})>5,000$ (National Marine Fisheries Service 2015). For each characteristic in turn, impulsiveness (coded 0 for non-impulsive and 1 for impulsive) was modeled as a function of "distance from source". These analyses were conducted within Generalized Linear Models (binomial error family) using a logit link function (Dobson 1990). Using a Wald's test (Hardin and Hilbe 2003), we examined whether "distance from source" had a significant effect on the probability of characteristics exceeding the above thresholds for impulsiveness. It should be noted that each signal type and location were modeled separately (i.e., seismic and pile driving signals were not pooled nor were pile driving signals at separate locations pooled). Further, given that the data consisted of observations (acoustic signals) collected close together in time, consecutive observations are likely to be correlated beyond the underlying processes included in the model, resulting in residual autocorrelation, which violates a key assumption of GLMs. Therefore, an investigation of temporal autocorrelation using the acf function within the $\mathrm{R}$ stats package (R Core Team, 2017) was carried out during the modeling. Where residual autocorrelation was present, each data set was reduced by selecting every $n$th observation, and the temporal autocorrelation re-assessed in plots of the acf function (Rooney et al. 1998). This was repeated with increasing values of $n$ until no residual autocorrelation was evident in the acf function plots; this effectively provided the maximum number of observations for modeling while minimizing residual autocorrelation (see Appendix S3 and S4).

\section{RESULTS}

\section{Pile driving}

The characteristics of each acoustic signal were successfully measured at ranges of between 0.5 and $40.1 \mathrm{~km}$ for the pile driving (Table 1). Signal duration for pile driving ranged from 0.06 to $1.27 \mathrm{~s}$ (mean $=0.30 ; 95 \%$ CIs $=0.07-0.83)$, rise time ranged from 2.77 to $495.00 \mathrm{~ms} \quad($ mean $=55.60 ; 95 \%$ CIs $=10.88-148.63)$, crest factor ranged from 8.46 to $18.30 \mathrm{~dB}$ (mean = $12.00 ; 95 \%$ CIs $=9.70-15.42$ ), and the quotient of peak pressure $(\mathrm{Pa})$ and pulse duration (s) ranged from 17.55 to $77,425.87 \mathrm{~Pa} / \mathrm{s}$ (mean $=6,866.21 ; 95 \% \mathrm{CIs}=40.09$ $28,772.64)$. Characteristics of signals from both sources exhibited marked variation with range (Fig. 1). Although characteristics exhibited wide variation throughout the range of the recordings, there were general decreases in peak pressure with range from source (Fig. 2), and general increases in signal duration (s), rise time of the signal (ms) and crest factor (dB) with range (Fig. 2).

For the majority of study area and characteristic combinations, distance from source had a significant effect on the probability of a sound being considered impulsive (Table 2 and Appendix S4). However, the coefficients of the effect of distance from source on the probability of a signal being considered impulsive varied depending on the characteristic modeled. Specifically, the mean probability of a signal duration being considered impulsive $(<1 \mathrm{~s}$ in duration) was $>0.9$ at ranges up to $33.1 \mathrm{~km}$ (Fig. 3). In contrast, rise time and the quotient of peak pressure (Pa) and pulse duration (s) indicated that signals should only be considered impulsive at much shorter ranges. The mean probability of the rise time being $<25 \mathrm{~ms}$ was $>0.9$ to ranges of 0.5 and $1.0 \mathrm{~km}$ and showed steep declines to $<0.5$ at ranges of approximately 1.2 and $2.5 \mathrm{~km}$ (Fig. 3) for the Moray Firth and Wash pile driving, respectively. The mean probability of the quotient of peak pressure $(\mathrm{Pa})$ and pulse duration (s) being considered impulsive $(>5,000 \mathrm{~Pa} / \mathrm{s})$ was $>0.9$ to ranges of approximately $3.4 \mathrm{~km}$ and showed steep declines to $<0.5$ at $3.5 \mathrm{~km}$ (Fig. 3). The mean probability of a crest factor being considered impulsive $(>15 \mathrm{~dB})$ was

TABLE 1. Summary of the values of the measured and derived characteristics for the acoustic signals from pile driving associated with the installation of wind turbine foundations in the Wash, England, and the Moray Firth, Scotland, and the seismic surveys in the Moray Firth, Scotland.

\begin{tabular}{lcccc}
\hline \hline Metric & Seismic & $\begin{array}{c}\text { Pile driving } \\
\text { (Wash) }\end{array}$ & $\begin{array}{c}\text { Pile driving } \\
\text { (Moray Firth) }\end{array}$ & Units \\
\hline $\begin{array}{l}\text { Crest factor } \\
\text { Peak }\end{array}$ & $14.8(2.2)$ & $14.0(1.1)$ & $10.3(0.8)$ & $\mathrm{dB}$ \\
$\begin{array}{l}\text { pressure/ } \\
\text { signal }\end{array}$ & $30.2(22.0)$ & $72.4(14.3)$ & $290.2(28.9)$ & $\mathrm{Pa} / \mathrm{s}$ \\
$\begin{array}{l}\text { duration } \\
\text { Peak } \\
\text { pressure }\end{array}$ & $25.8(0.9)$ & $54.2(6.8)$ & $94.2(7.8)$ & $\mathrm{Pa}$ \\
$\begin{array}{l}\text { Rise time } \\
\text { Signal } \\
\text { duration }\end{array}$ & $75.5(0.3)$ & $95.7(33.6)$ & $134.3(69.8)$ & $\mathrm{ms}$ \\
\hline
\end{tabular}

Notes: For comparative purposes here, acoustic signals have been limited to those recorded between 8 and $10 \mathrm{~km}$ from the source. Values are mean with SD in parentheses. 

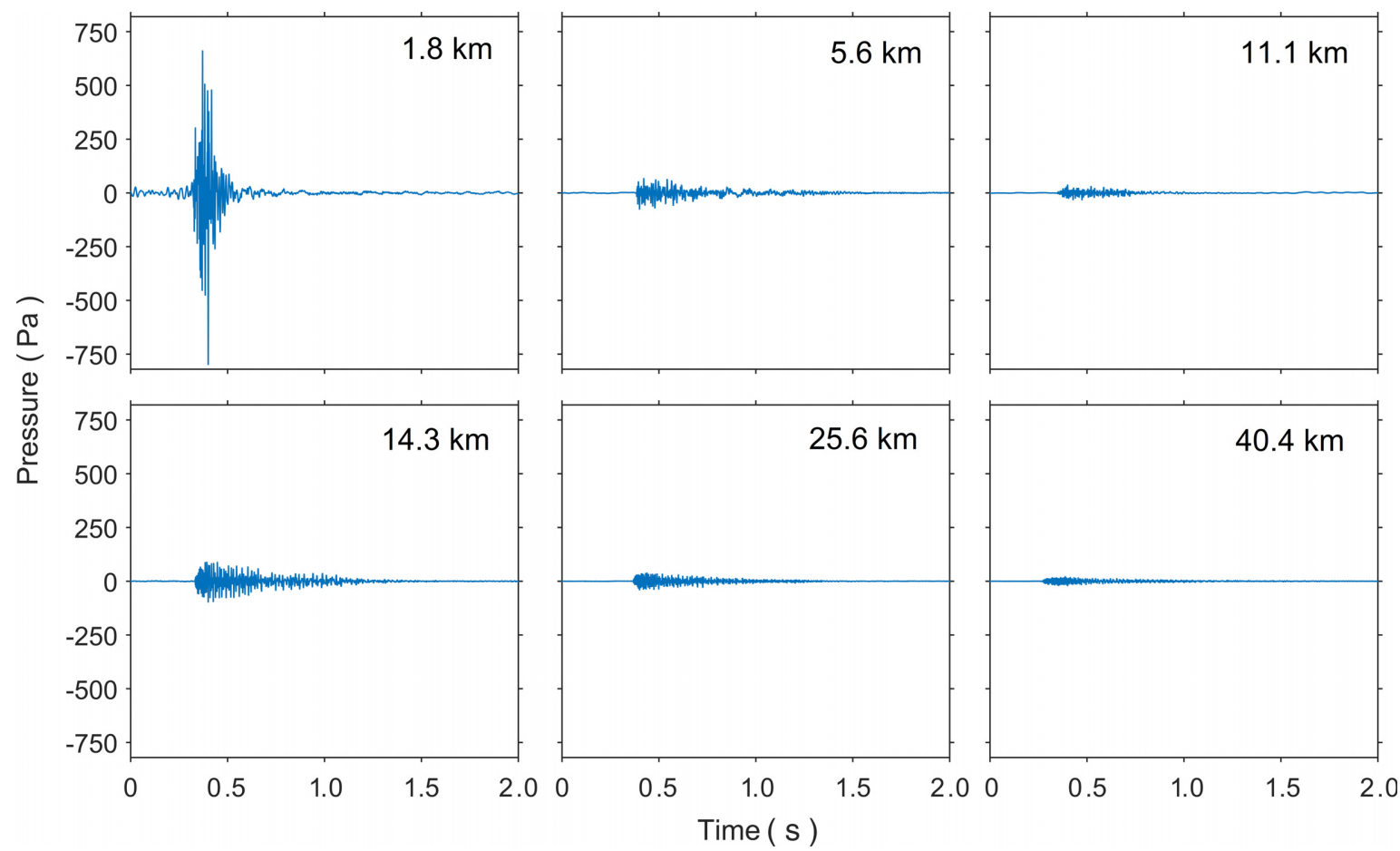

FIG. 1. Example of the variation in waveforms of the acoustic signals from seismic surveys at a series of recording ranges (from 1.8 to $40.4 \mathrm{~km}$ ). The $x$-axis shows time and the $y$-axis is pressure.

$<0.5$ throughout the entire range of the Wash pile driving data. It should also be noted that the models of signal duration and quotient of peak pressure and pulse duration for the pile driving in The Wash were not run because signal durations were all $<1 \mathrm{~s}$ or had a quotient of $<5,000 \mathrm{~Pa} / \mathrm{s}$. Similarly, the model of crest factor for the pile driving in the Moray Firth was not run because all signals had crest factors $<15 \mathrm{~dB}$.

\section{Seismic survey}

The characteristics of the seismic signals were measured at ranges of between 1.6 and $61.8 \mathrm{~km}$ (Table 1). Signal duration ranged from 0.09 to $1.88 \mathrm{~s}$ (mean $=0.69$ $95 \%$ CIs $=0.12-1.50)$, rise time ranged from 0.92 to $398.74 \mathrm{~ms} \quad($ mean $=66.48 ; 95 \% \quad$ CIs $=15.18-152.80)$, crest factor ranged from 7.08 to $20.68 \mathrm{~dB}$ (mean = $12.79 ; 95 \% \mathrm{CIs}=8.78-17.07$ ), and the quotient of peak pressure and pulse duration ranged from 1.57 to 9,875.98 Pa/s $\quad($ mean $=1030.89 ; \quad 95 \% \quad$ CIs $=7.16-$ 5.432.49). Signal characteristics exhibited similar patterns to the pile driving; there was a general decrease in peak pressure with range, and general increases in signal duration, rise time of the signal and crest factor with range, although each exhibited wide variation throughout the range of the recordings (Fig. 2). As with pile driving, for the majority of characteristics, distance from source had a significant effect on the probability of a sound being considered impulsive (Table 2 and
Appendix S4). The coefficients of the effect of distance from source on the probability of a signal being considered impulsive varied depending on the characteristic modeled. Specifically, the mean probability of a signal duration being $<1 \mathrm{~s}$ was $>0.9$ at ranges up to $6.7 \mathrm{~km}$ and declined to $<0.5$ beyond $46 \mathrm{~km}$ (Fig. 3). The mean probability of the rise time being $<25 \mathrm{~ms}$ was $<0.3$ throughout the entire range for seismic surveys (Fig. 3). The mean probability of the quotient of peak pressure and pulse duration being $>5,000 \mathrm{~Pa} / \mathrm{s}$ was $>0.9$ to ranges of $1.6 \mathrm{~km}$ and showed a steep decline to $<0.5$ at $1.9 \mathrm{~km}$ (Fig. 3). The mean probability of a crest factor $>15 \mathrm{~dB}$ remained $<0.5$ throughout the entire range for seismic surveys.

\section{DisCUSSION}

This study utilized existing underwater acoustic recordings made during offshore seismic surveys and pile driving activities to explore how impulsive acoustic signals change as they propagate from source. Our results show that the impulsive characteristics of underwater sound change markedly as a result of propagation and suggest that only a proportion of received signals would be described as impulsive based on previous definitions of impulsive noise.

Studies of terrestrial mammals generally conclude that, at comparable sound levels, impulsive noise is more hazardous than continuous noise with respect to hearing 

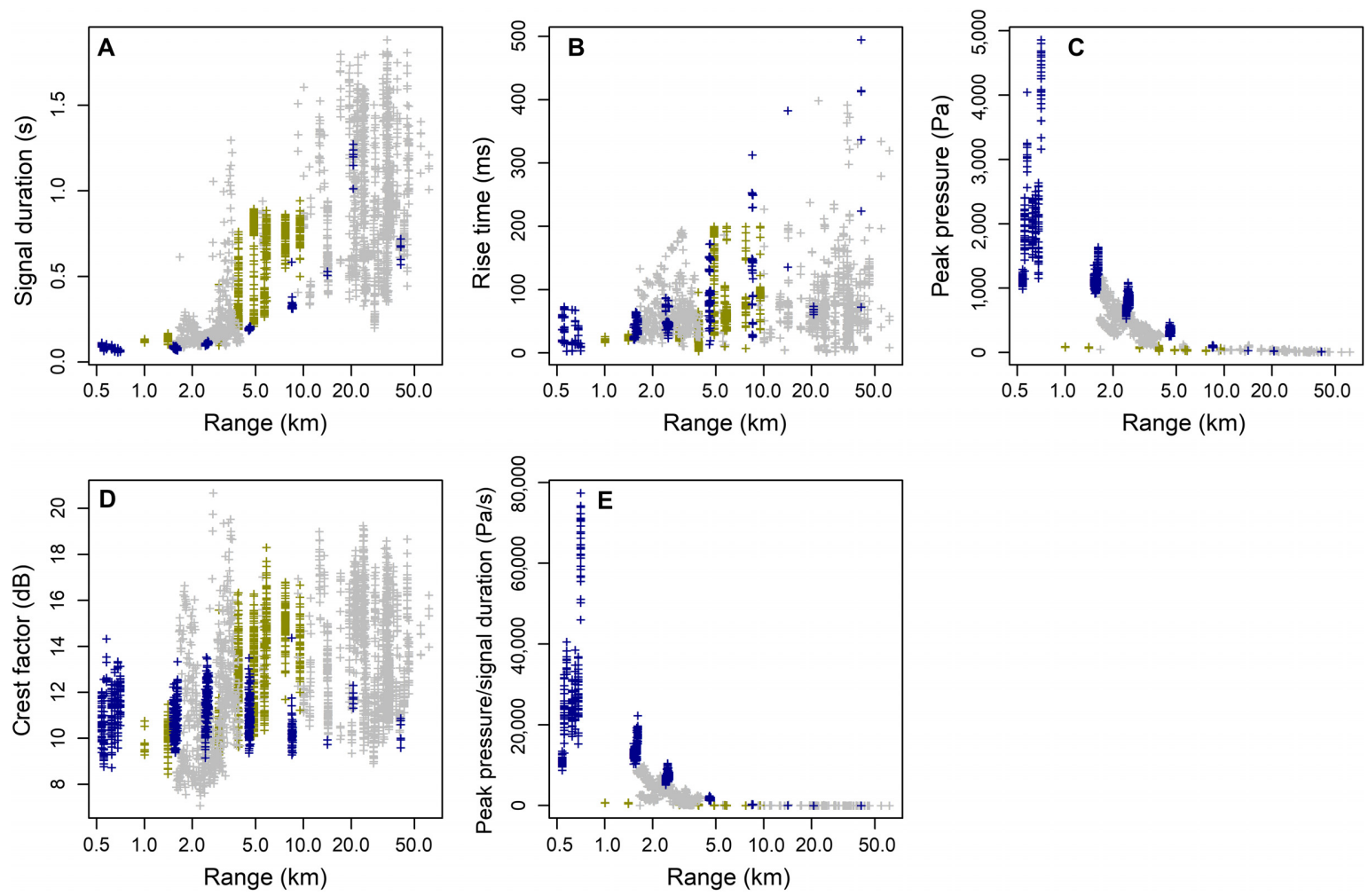

FIG. 2. Plot of the characteristics of the signals produced during pile driving and a seismic survey plotted as a function of range from the source. (A) Signal duration, (B) rise time, (C) peak pressure, (D) crest factor, and (E) the quotient of peak pressure and signal duration. The points are color coded to show the source of the signals (gray, seismic; blue, pile driving in the Moray Firth; yellow, pile driving in The Wash).

TABLE 2. Summary of the binomial models describing the relationships between distance from source and the probability of a signal being impulsive for acoustic signals from pile driving associated with the installation of wind turbine foundations in the Wash, England, and the Moray Firth, Scotland, and seismic surveys in the Moray Firth, Scotland. Note that cells containing a dash represent models that were not run.

\begin{tabular}{|c|c|c|c|c|c|c|}
\hline Characteristic and source & Location & Intercept & $\beta$ & $\chi^{2}$ & $\mathrm{df}$ & $P$ \\
\hline \multicolumn{7}{|l|}{ Crest factor } \\
\hline Seismic & Moray Firth & -1.759 & 2.7635 & 3.7 & 1 & 0.0561 \\
\hline Pile driving & Moray Firth & - & - & - & - & - \\
\hline Pile driving & Wash & -3.420 & 0.0003 & 6.5 & 1 & 0.0108 \\
\hline \multicolumn{7}{|l|}{ Peak pressure/signal duration } \\
\hline Seismic & Moray Firth & 6.925 & -0.0037 & 7.7 & 1 & 0.0056 \\
\hline Pile driving & Moray Firth & 82.871 & -0.0235 & 0.0 & 1 & 0.9994 \\
\hline Pile driving & Wash & - & - & - & - & - \\
\hline \multicolumn{7}{|l|}{ Rise time } \\
\hline Seismic & Moray Firth & -2.805 & 3.6480 & 6.1 & 1 & 0.0135 \\
\hline Pile driving & Moray Firth & 2.918 & -0.0024 & 148.7 & 1 & $<0.0001$ \\
\hline Pile driving & Wash & 1.989 & -0.0008 & 6.3 & 1 & 0.0118 \\
\hline \multicolumn{7}{|l|}{ Signal duration } \\
\hline Seismic & Moray Firth & 2.564 & -5.478 & 12.5 & 1 & 0.0004 \\
\hline Pile driving & Moray Firth & 6.140 & -0.0001 & 4.25 & 1 & 0.0393 \\
\hline Pile driving & Wash & - & - & - & - & - \\
\hline
\end{tabular}

Notes: The table shows the model coefficients and significance based on a Wald's Test (Hardin and Hilbe 2003). Parameters include crest factor $(\mathrm{dB})$, peak pressure/signal duration $(\mathrm{Pa} / \mathrm{s})$, rise time $(\mathrm{ms})$, and the duration of the signal (s). It should be noted that the model of Peak pressure/signal duration for pile driving signals in the Moray Firth exhibited a step change relationship with range from source so values for $\chi^{2}$ and $P$ should be interpreted with caution. 

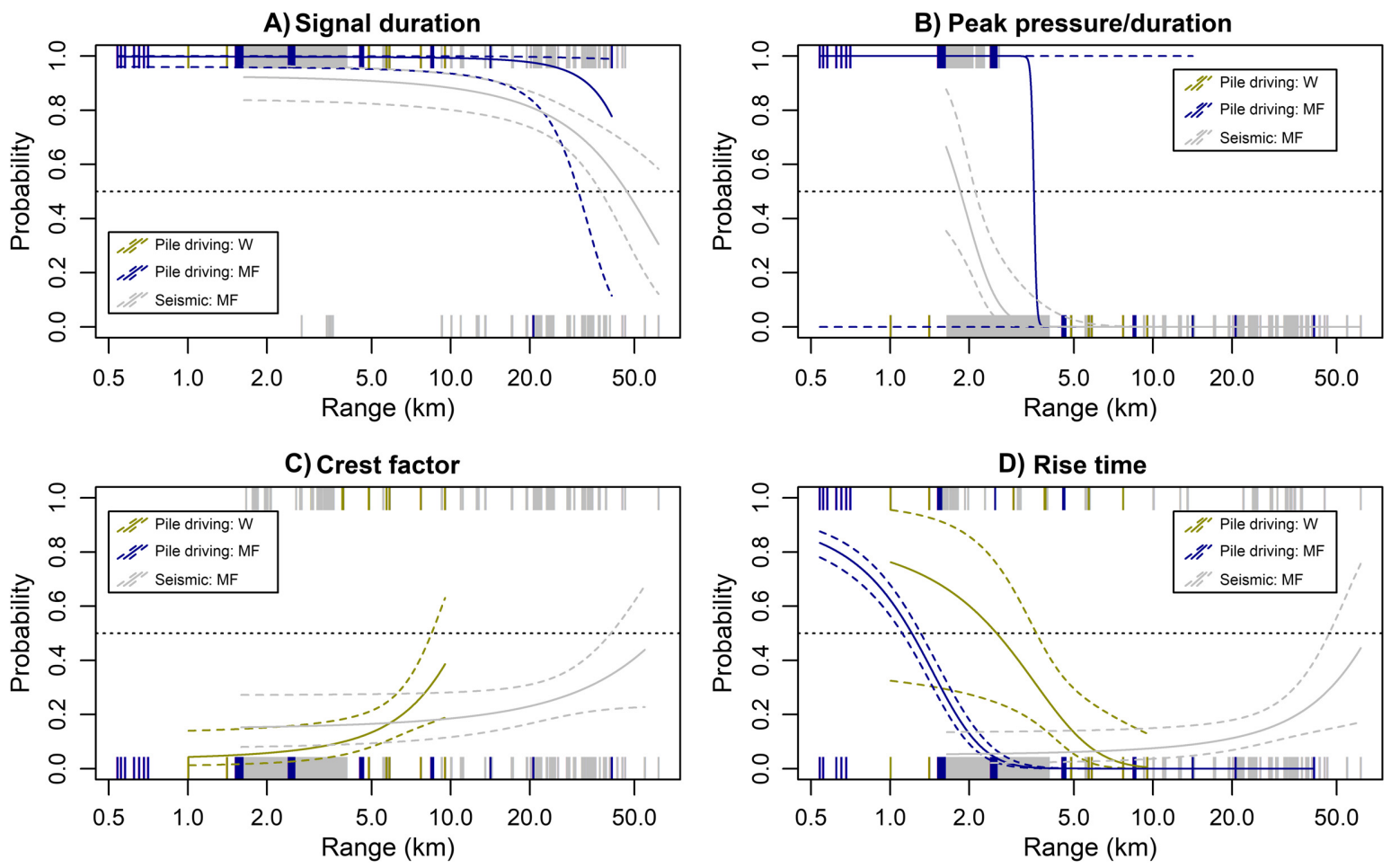

FIG. 3. Modeled functions describing the probability of a signal being defined as "impulsive" based on (A) the signal duration being $<1 \mathrm{~s}$, (B) the quotient of peak pressure and signal duration exceeding 5,000 $\mathrm{Pa} / \mathrm{s},(\mathrm{C})$ the crest factor exceeding $15 \mathrm{~dB}$, and (D) the rise time being $<25 \mathrm{~ms}$. The lines represent the modeled fits and their $95 \%$ confidence intervals. The points and lines are color coded to show the source of the signals (gray, seismic; blue, pile driving in the Moray Firth; yellow, pile driving in The Wash). It should be noted that the models of signal duration and peak pressure/duration for the pile driving in The Wash did not converge because all signals were $<1 \mathrm{~s}$ or had a peak pressure/duration value of $<5,000$. Similarly, the model of crest factor for the pile driving in the Moray Firth did not converge because all signals had crest factors less than $15 \mathrm{~dB}$.

damage (Sulkowski and Lipowczan 1982, Dunn et al. 1991). In marine mammals, exposure to sounds described as impulsive sounds has been shown to cause TTS in several species, including beluga whale (Delphinapterus leucas; Finneran et al. 2002), bottlenose dolphins (Finneran et al. 2015), and harbor porpoises (Kastelein et al. 2015). In contrast, no TTS was detected in related studies of a beluga whale and bottlenose dolphins exposed to impulsive underwater sounds but with characteristics resembling distant (ranges from 1.5 to $55.6 \mathrm{~km}$ ) signatures of underwater explosions (Finneran et al. 2000); however, with respect to the impulsive characteristics of these exposures, differences between the studies (Finneran et al. 2000, 2002, 2015, Kastelein et al. 2015) may be due to other factors such as exposure levels, duty cycle, experimental setup, and hearing sensitivity of the test subjects and species.

Despite recognition that the relative risk of underwater noise for marine mammals depends partly upon whether it is impulsive or non-impulsive, no single mathematical definition currently exists for impulsive sound in this context. More generally, impulsive sounds underwater have been described as those with a rapid rise time, short signal duration (Southall et al. 2007), high peak pressure (Thiery 1987), and high crest factor (Starck and Pekkarinen 1987). However, efforts to assess the risks of particular noise sources remain constrained, both by the absence of agreed quantitative definitions of impulsive sound and an understanding of how these characteristics vary with distance from source.

Our analysis of recordings from three different broadscale acoustic studies demonstrated how a range of impulsive characteristics varied as a function of distance from source. The greatest change in the majority of metrics occurred between 0.5 and $10 \mathrm{~km}$ of the source. This was particularly pronounced for peak pressure, signal duration, and rise time (Fig. 2). Peak pressure decreased rapidly to remain below $100 \mathrm{~Pa}$ from $4.7 \mathrm{~km}$. Signal duration was generally lower in the recordings closest to the source and increased rapidly to plateau from approximately $10 \mathrm{~km}$ from source. Similarly, although short rise times were present throughout the data, variation was generally lower closest to the source and was more variable from approximately $5-10 \mathrm{~km}$. In contrast, there appeared to be a relatively poor relationship between range and crest factor with this metric exhibiting a high degree of variation throughout the data. 
These results have important implications for the assessment of auditory damage in marine mammals and the subsequent management of underwater noise. Recent guidance for assessing the impacts of anthropogenic sound on marine mammal hearing investigated changes in impulsive characteristics as sound propagates from source (National Marine Fisheries Service 2015). The final guidance follows previous approaches (ANSI 1986, NIOSH 1998) and defines impulsive signals as being transient, brief (less than $1 \mathrm{~s}$ ), broadband, and typically consisting of high peak pressure with rapid rise time and rapid decay. During the development of the guidance, a transition threshold (National Marine Fisheries Service 2015) was also proposed, which used the quotient of peak pressure signal duration as a surrogate for rise time. Through an investigation of the literature, it was initially suggested that a threshold at which most impulsive sound sources begin to transition to having physical characteristics less likely to result in auditory injury generally occurs at ranges of $\sim 3 \mathrm{~km}$ from source. However, due to a lack of evidence to support the transition range approach, the authors removed this approach from the final guidance (National Marine Fisheries Service 2018, Southall et al. 2019). Nevertheless, they highlight the need to understand how the characteristics of a sound that makes it injurious change under various propagation conditions to develop appropriate thresholds in future. With particular reference to this impulsive/non-impulsive transition, the quotient calculated here varied from 17 to $77,496 \mathrm{~Pa} / \mathrm{s}$ for pile driving signals and from 4 to $10,330 \mathrm{~Pa} / \mathrm{s}$ for seismic signals. A total of $503(36 \%)$ of the pile driving signals and $133(6 \%)$ of the seismic signals exceeded the draft threshold $(5,000)$ and all occurred relatively close to the source, being within $2.54 \mathrm{~km}$ of the pile driving and $2.60 \mathrm{~km}$ of the seismic air guns. At least from a range perspective, this provides support to the initial conclusion that the quotient of peak pressure and signal duration generally exceeds $5,000 \mathrm{~Pa} / \mathrm{s}$ within $2-3 \mathrm{~km}$ from the source for pile driving and seismic signals (National Marine Fisheries Service 2015).

Based on the argument that the transition threshold (National Marine Fisheries Service 2015) was investigated as a surrogate for rise time, the modeling results for rise time also broadly support the conclusions that rise time increases with range from the source. Although short rise times were present throughout the data, the mean probability of a rise time being $<25 \mathrm{~ms}$ was $>0.9$ to ranges of 0.5 and $1.0 \mathrm{~km}$ and showed steep declines to $<0.5$ at ranges of approximately 1.2 and $2.5 \mathrm{~km}$ for the Moray Firth and Wash pile driving, respectively. Conversely, the mean probability of the rise time being $<25 \mathrm{~ms}$ for seismic survey signals was $<0.3$ throughout the entire range. The disparity between these results may partly be explained by a lack of data for seismic signals at relatively close ranges (within $1.6 \mathrm{~km}$ ) where short rise times were predicted in the pile driving data. It is also important to consider the caveats described below when interpreting the rise time results; for example, the observed increase in the probability of rise times being $<25 \mathrm{~ms}$ with distance from source in the seismic data may partly be an artefact of low signal-to-noise ratios at large distances, which can make accurate measurement of rise time difficult.

Previous studies have also considered defining impulsive sounds as those where the signal duration is $<1 \mathrm{~s}$ (Southall et al. 2007, National Marine Fisheries Service 2015, 2018, Southall et al. 2019). Using this approach, the majority of both the seismic $(1,666 ; 76.9 \%)$ and pile driving signals $(1,397 ; 99.6 \%)$ that we recorded would be considered impulsive. Our recordings are also within the range of previous measurements of duration from seismic and pile driving signals; for example, Greene and Richardson (1988) measured durations of $0.25-0.75 \mathrm{~s}$ for seismic signals at 3-4 km from the air guns, and Blackwell (2005) measured durations of between $\sim 0.1$ and $\sim 0.2 \mathrm{~s}$ for signals between 63 and 1,100 $\mathrm{m}$ from pile driving.

Building upon studies of human auditory damage, Starck and Pekkarinen (1987) proposed that signals should be considered as impulsive if they have crest factors that exceed $15 \mathrm{~dB}$. We found that $429(23 \%)$ of seismic and $112(8 \%)$ of pile driving signals had crest factors exceeding $15 \mathrm{~dB}$. However, there was no clear relationship between range from source and crest factor, in contrast to all other impulsive metrics. Compared to the other acoustic characteristics that indicate that the impulsiveness of the signals decreases with range from source, the results of the GLMs describing changes in crest factor suggest that the probability of a crest factor exceeding $15 \mathrm{~dB}$ increases with range from source for both pile driving and seismic signals. Although seemingly counterintuitive, it is important to highlight that such a pattern may simply be an artifact of complex interference patterns, the large variation in measured crest factors, or that the ranges of the recordings to the source (closest $500 \mathrm{~m}$ ) were already further than those where high crest factors might be predicted. It is therefore recommended that further data are collected at ranges closer to source to formally address this limitation.

More generally, it should be highlighted that the acoustic characteristics measured in the current study may suffer from a degree of measurement error, particularly for signals recorded at relatively large distances from the source. For example, low signal-to-noise ratios may result in difficulties in determining signal onset and end for measuring signal duration or rise time or any of the other metrics derived from these. Further, the derivation of rise time and crest factor here were based on the timing and amplitude of peak pressure within the signal, respectively. Although this may be an adequate approach to describe rise time in signals with a clear onset and high peak pressure, it risks being inadequate where complex multipath propagation and interference patterns result in spurious high peak pressures relatively far into the signal. Similarly, crest factors have the potential to be influenced by uncertainties in signal 
duration at low signal-to-noise ratios and as a result of complex propagation; this may be particularly apparent for signals recorded at large distances from the source. Interpretation of the results, particularly those at large distances, should therefore bear these caveats in mind.

Although a number of the acoustic characteristics of the signals analysed here showed clear patterns with range from the source, the recordings are all from relatively shallow water coastal environments with relatively flat seabed topography. Further, changes in the impulsive characteristics of signals are likely to be environment specific, and seismic surveys or pile driving carried out in different habitats (e.g., nearshore or deep water) may therefore exhibit different propagation patterns. This highlights the need for similar studies in contrasting environments. In the current study, we only tested one single variable (range from source) in the GLMs. The measured variation in the relationship with range in each model therefore represents variation due to either measurement error (as described above) or variation in source characteristics or propagation conditions between measurements. A number of other key determinants that were not available for testing in the models here may explain variation between and within study areas; these are likely to include dynamic environmental variables (e.g., sea state or tidal height), topographical variables (e.g., sediment depth and type), or variables related to the source characteristics (e.g., piling blow energy or air gun power); it would therefore be useful to explore the effects of these in more detail in future studies. Nevertheless, in the meantime, our results provide useful insights into the behavior of propagating impulsive sounds in shallow water environments that are typically used for offshore wind turbines and the majority of offshore oil exploration (Pinder 2001).

In summary, based on the measured characteristics of the seismic and pile driving signals, it seems clear that, at least a proportion of the recorded signals should be defined as impulsive based on previous descriptors (Starck and Pekkarinen 1987, Thiery 1987, Southall et al. 2007, National Marine Fisheries Service 2015). However, considering each characteristic in isolation as a criterion for the impulsive nature of a sound is misleading as it is likely that a complex interaction of these, and many other unrelated parameters such as duty cycle, recovery periods, absolute SPL and SELs will strongly affect the risk of hearing damage.

\section{Implications for the management of anthropogenic noise}

From an applied perspective, the results presented here are important to consider when predicting the effects of activities that produce impulsive sound on marine mammals. In practice, during an environmental impact assessment of a marine activity, an analysis can be carried out at where the spatial distribution of marine mammals is compared to spatial predictions of the acoustic levels (Thompson et al. 2013b). These data are then integrated with available data on the potential impacts of noise to estimate the number of individuals that may be impacted, which can then be compared to biological or regulatory impact thresholds.

Following regulatory guidance in many countries, (JNCC 2010, Government of South Australia 2012, Marine Scotland 2014, NPWS 2014) the most commonly applied thresholds are currently those developed by Southall et al. (2007). Within such assessments, changes in the acoustic characteristics with propagation are generally limited to measures related to sound intensity (Thompson et al. 2013b, Hastie et al. 2015, Hermannsen et al. 2015); other characteristics such as those measured in the current study are generally not considered. As a result, it is typically assumed that the broad characteristics of a signal (impulsive or non-impulsive) will remain constant throughout its propagation range. Given that published thresholds for PTS onset as a result of exposure to impulsive signals are lower than the nonimpulsive thresholds in all species groups (Southall et al. 2007), there is therefore potential that the risk of auditory damage may be overestimated in cases where impulsive signals become non-impulsive with propagation at ranges lower than those where cSELs are predicted to exceed impulsive auditory damage thresholds. However, it is important to note that any reduced risk that originates from the impulse becoming "non-impulsive" may partly be counteracted by the increase in signal duration. This is because a longer signal will have a relatively higher SEL than what one would expect from predictions based on the signal characteristics measured close to the source. It is also important to bear in mind that a range of other factors such as duty cycle and the respective recovery periods between signals will also likely influence the risk of hearing damage from repetitive sounds such as those from pile driving and seismic surveys (Nilsson 1991). We therefore recommend that further TTS and physiological studies of marine mammals are carried out to explicitly determine which acoustic parameters of "impulsiveness" are the best predictors of injury risk.

This has clear implications for the management of underwater noise and the regulation of anthropogenic activities in the marine environment. This can be particularly challenging for regulators when balancing the costs and benefits of activities, such as offshore windfarm construction, which are being used to address other environmental policy goals (Merchant 2019). In terms of the practical application of the results, future impact assessments would benefit from a recognition of changes in the acoustic characteristics of impulsive sounds when predicting the likelihood of auditory damage in marine mammals from impulsive sounds; formal consideration, potentially through approaches such as time-domain modeling of pulse propagation (Farcas et al. 2016), could be given to ranges where signals may lose their more injurious characteristics. This could also be used to inform the development of strategies to monitor the 
potential impacts of noise; for example, in cases where the measurement and reporting of sound levels during activities are required as part of consenting conditions (e.g., Andersson et al. 2016), consideration could be made about the reporting of additional acoustic metrics such as those measured here in order to characterize risk. Further, predicted signal transition (from impulsive to non-impulsive) distances may be used to inform whether technical mitigation measures (e.g., noise reduction measures such as bubble curtains) or operational mitigation measures (e.g., passive acoustic monitoring or visual observers to detect animals in real time) may be effective at reducing risk.

\section{ACKNOWLEDGMENTS}

This work was funded as part of the Department of Energy and Climate Change's Offshore Energy Strategic Environmental Assessment programme, with additional resources from the National Capability funding from the Natural Environment Research Council to the Sea Mammal Research Unit (grant no. SMRU1001). Recordings of piling and seismic noise from the Moray Firth were collected with the support of the Department of Energy and Climate Change, Scottish Government, Oil and Gas UK Ltd., COWRIE, EU DOWNVInD project, Talisman Energy (UK) Ltd. and Scottish \& Southern Energy. Thanks also to Helen Bailey and Keith Needham for making field recordings, Francesca Marubini for comments and suggestions on drafts of the manuscript, and to Stephen Robinson at the National Physics Laboratory and Douglas Gillespie at the Sea Mammal Research Unit for discussions and guidance about acoustic analyses.

\section{Literature Cited}

Andersson, M. H., S. Andersson, J. Ahlsén, B. L. Andersson, J. Hammar, L. K. G. Persson, J. Pihl, P. Sigray, and A. Wikström. 2016. A framework for regulating underwater noise during pile driving. A technical Vindval report. Swedish Environmental Protection Agency, Stockholm, Sweden.

ANSI. 1986. Methods of measurement for impulse noise (ANSI S12.7-1986). Acoustical Society of America, New York, New York, USA

Bailey, H., B. Senior, D. Simmons, J. Rusin, G. Picken, and P. M. Thompson. 2010. Assessing underwater noise levels during pile-driving at an offshore windfarm and its potential effects on marine mammals. Marine Pollution Bulletin 60:888-897.

Blackwell, S. B. 2005. Underwater measurements of pile-driving sounds during the Port MacKenzie dock modifications, 1316 August 2004. Report from Greeneridge Sciences, Goleta, California, and LGL Alaska Research Associates, Anchorage, Alaska, in association with HDR Alaska, Anchorage, Alaska, for Knik Arm Bridge and Toll Authority, Anchorage, Alaska, Department of Transportation and Public Facilities, Anchorage, Alaska, and Federal Highway Administration, Juneau, Alaska, USA.

Buck, K. 1982. Influence of different presentation patterns of a given noise dose on hearing in Guinea pig. Scandinavian Audiology Supplementum 16:83-87.

Dobson, A. J. 1990. An introduction to generalized linear models. Chapman and Hall, London, UK.

Dunn, D. E., R. R. Davis, C. J. Merry, and J. R. Franks. 1991. Hearing loss in the chinchilla from impact and continuous noise exposure. Journal of the Acoustical Society of America 90:1979-1985.

Farcas, A., C. F. Powell, R. C. Faulkner, G. D. Hastie, P. M. Thompson, and N. D. Merchant. 2016. Time-domain modelling of underwater transient noise for environmental risk assessment. Journal of the Acoustical Society of America 140:3072.

Finneran, J. J., C. E. Schlundt, D. A. Carder, J. A. Clark, J. A. Young, J. B. Gaspin, and S. H. Ridgway. 2000. Auditory and behavioral responses of bottlenose dolphins (Tursiops truncatus) and a beluga whale (Delphinapterus leucas) to impulsive sounds resembling distant signatures of underwater explosions. Journal of the Acoustical Society of America 108:417431.

Finneran, J. J., C. E. Schlundt, R. Dear, D. A. Carder, and S. H. Ridgway. 2002. Temporary shift in masked hearing thresholds in odontocetes after exposure to single underwater impulses from a seismic watergun. Journal of the Acoustical Society of America 111:2929-2940.

Finneran, J. J., C. E. Schlundt, B. K. Branstetter, J. S. Trickey, V. Bowman, and K. Jenkins. 2015. Effects of multiple impulses from a seismic air gun on bottlenose dolphin hearing and behavior. Journal of the Acoustical Society of America 137:1634-1646.

Fletcher, J. L. 1970. Temporary threshold shift recovery from impulse and steady state noise exposure. Journal of the Acoustical Society of America 48:96.

Gordon, J., D. Gillespie, J. Potter, A. Frantzis, M. P. Simmonds, R. Swift, and D. Thompson. 2004. A review of the effects of seismic surveys on marine mammals. Journal of Marine Technology 37:16-34.

Government of South Australia. 2012. Underwater Piling Noise Guidelines. Report by the Department of Planning, Transport and Infrastructure Version 1, Adelaide, Australia.

Greene, C. R., Jr., and W. J. Richardson. 1988. Characteristics of marine seismic survey sounds in the Beaufort Sea (Canada, USA). Journal of the Acoustical Society of America 83:22462254.

Hamernik, R. P., J. H. Patterson, and R. J. Salvi. 1987. The effect of impulse intensity and the number of impulses on hearing and cochlear pathology in the chinchilla. Journal of the Acoustical Society of America 81:1118-1129.

Hardin, J. W., and J. M. Hilbe. 2003. Generalized estimating equations. Chapman and Hall/CRC Press, London, UK.

Hastie, G. D., D. J. F. Russell, B. J. McConnel, S. Moss, D. Thompson, and V. M. Janik. 2015. Sound exposure in harbour seals during the installation of an offshore wind farm: predictions of auditory damage. Journal of Applied Ecology 52:631-640.

Henderson, D., and R. P. Hamernik. 1986. Impulse noise: Critical review. Journal of the Acoustical Society of America 80:569-584.

Hermannsen, L., J. Tougaard, K. Beedholm, J. Nabe-Nielsen, and P. T. Madsen. 2015. Characteristics and propagation of airgun pulses in shallow water with implications for effects on small marine mammals. PLoS ONE 10:e0133436.

JNCC. 2010. The protection of marine European Protected Species from injury and disturbance. Draft guidance for the marine area in England and Wales and the UK offshore marine area. JNCC, Natural England and Countryside Council for Wales. https://assets.publishing.service.gov.uk/government/ uploads/system/uploads/attachment_data/file/681834/Protection_ Marine EP Injury Disturbance.pdf

Kastelein, R. A., R. Gransier, M. A. T. Marijit, and L. Hoek. 2015. Hearing frequency thresholds of harbor porpoises (Phocoena phocoena) temporarily affected by played back 
offshore pile driving sounds. Journal of the Acoustical Society of America 137:556-564.

Kryter, K. D. 1994. The handbook of hearing and the effects of noise: physiology, and public health. McGraw-Hill, New York, New York, USA.

Luz, G. A., and D. C. Hodge. 1970. Recovery from impulsenoise induced TTS in monkeys and men: a descriptive model. Journal of the Acoustical Society of America 49:1770-1777.

Marine Scotland. 2014. The protection of Marine European Protected Species from injury and disturbance: Guidance for Scottish Inshore Waters. Report prepared by Scottish Government in partnership with Scottish Natural Heritage Commissioned Report No. 441, Edinburgh, UK.

Merchant, N. D. 2019. Underwater noise abatement: Economic factors and policy options. Environmental Science and Policy 92:116-123.

National Marine Fisheries Service. 2015. DRAFT guidance for assessing the effects of anthropogenic sound on marine mammal hearing: underwater acoustic thresholds levels for onset of permanent and temporary threshold shifts. U.S. Department of Commerce, NOAA Technical Memorandum.

National Marine Fisheries Service. 2018. Technical guidance for assessing the effects of anthropogenic sound on marine mammal hearing: underwater acoustic thresholds for onset of permanent and temporary threshold shifts. NOAA Technical Memorandum NOAA Technical Memorandum NMFSOPR-55. U.S. Department of Commerce, NOAA, Washington, D.C., USA

Nilsson, R. 1991. The effect of rest periods on temporary threshold shift (TTS) after noise exposure. Journal of Sound and Vibration 151:437-440.

NIOSH. 1998. Criteria for a recommended standard: Occupational noise exposure. United States Department of Health and Human Services, Cincinnati, Ohio, USA.

NPWS. 2014. Guidance to manage the risk to marine mammals from man-made sound sources in Irish waters. Guidance Document by the Department of Arts, Heritage and the Gaeltacht. https://www.npws.ie/sites/default/files/general/Under water $\% 20$ sound $\% 20$ guidance_Jan $\% 202014$.pdf

Pinder, D. 2001. Offshore oil and gas: global resource knowledge and technological change. Ocean and Coastal Management 44:579-600.
R Core Team. 2017. R: A language and environment for statistical computing. R Foundation for Statistical Computing, Vienna, Austria. http://www.R-project.org/

Rooney, S. M., A. Wolfe, and T. J. Hayden. 1998. Autocorrelated data in telemetry studies: time to independence and the problem of behavioural effects. Mammal Review 28:89-98.

Southall, B. L., et al. 2007. Marine mammal noise exposure criteria: Initial scientific recommendations. Aquatic Mammals $33: 411-521$

Southall, B. L., J. J. Finneran, C. Reichmuth, P. E. Nachtigall, D. R. Ketten, A. Bowles, and Tyack, P. L. . 2019. Marine mammal noise exposure criteria: Updated scientific recommendations for residual hearing effects. Aquatic Mammals 45(2):125-232. https://doi.org/10.1578/AM.45.2.2019. 125

Starck, J., and J. Pekkarinen. 1987. Industrial impulse noise: Crest factor as an additional parameter in exposure measurements. Applied Acoustics 20:263-274.

Sulkowski, W. J., and A. Lipowczan. 1982. Impulse noise induced hearing loss in drop forge operators and the energy concept. Noise Control Engineering 18:24-29.

Thiery, L. 1987. Characterization of industrial impulse noises by discriminant analysis. Journal of Sound and Vibration 113:404-406

Thompson, P. M., K. L. Brookes, I. M. Graham, T. R. Barton, K. Needham, G. Bradbury, and N. D. Merchant. 2013a. Short-term disturbance by a commercial two-dimensional seismic survey does not lead to long-term displacement of harbour porpoises. Proceedings of the Royal Society B 280: 20132001.

Thompson, P. M., G. D. Hastie, J. Nedwell, R. Barham, K. L. Brookes, L. S. Cordes, H. Bailey, and N. McLean. 2013b. Framework for assessing impacts of pile-driving noise from offshore wind farm construction on a harbour seal population. Environmental Impact Assessment Review 43:73-85.

Ward, W. D., A. Glorig, and D. L. Sklar. 1959. Temporary shift from octave-band noise: Applications to damage-risk criteria. Journal of the Acoustical Society of America 41:522-528.

Yost, W. A. 2000. Fundamentals of hearing: an introduction. Fourth edition. Elsevier Science \& Technology Books, Leiden, The Netherlands.

\section{SUPPORTING INFORMATION}

Additional supporting information may be found online at: http://onlinelibrary.wiley.com/doi/10.1002/eap.1906/full

\section{Data Availability}

Data are available on the Dryad Digital Repository: https://doi.org/10.5061/dryad.qg41t6k 